\author{
Turkuler Ozgumus \\ Department of Mechanical Engineering, \\ Izmir Institute of Technology, \\ Urla, Izmir 35430, Turkey \\ e-mail: turkulerozgumus@iyte.edu.tr \\ Moghtada Mobedi ${ }^{1}$ \\ Department of Mechanical Engineering, \\ Izmir Institute of Technology, \\ Urla, Izmir 35430, Turkey \\ e-mail: moghtadamobedi@iyte.edu.tr
}

\section{Effect of Pore to Throat Size Ratio on Interfacial Heat Transfer Coefficient of Porous Media}

\begin{abstract}
In this study, the effects of pore to throat size ratio on the interfacial heat transfer coeffcient for a periodic porous media containing inline array of rectangular rods are investigated, numerically. The continuity, Navier-Stokes, and energy equations are solved for the representative elementary volume (REV) of the porous media to obtain the microscopic velocity and temperature distributions in the voids between the rods. Based on the obtained microscopic temperature distributions, the interfacial convective heat transfer coefficients and the corresponding Nusselt numbers are computed. The study is performed for pore to throat size ratios between 1.63 and 7.46, porosities from 0.7 to 0.9 , and Reynolds numbers between 1 and 100. It is found that in addition to porosity and Reynolds number, the parameter of pore to throat size ratio plays an important role on the heat transfer in porous media. For the low values of pore to throat size ratios (i.e., $\beta=1.63)$, Nusselt number increases with porosity while for the high values of pore to throat size ratios (i.e., $\beta=7.46$ ), the opposite behavior is observed. Based on the obtained numerical results, a correlation for the determination of Nusselt number in terms of porosity, pore to throat size ratio, Reynolds and Prandtl numbers is proposed. [DOI: $10.1115 / 1.4028764]$
\end{abstract}

Keywords: porous media, interfacial heat transfer coefficient, pore to throat size ratio

\section{Introduction}

The mechanism of heat and fluid flow in porous media is complex due to the existence of different phases and it is difficult to obtain the exact temperature, velocity, and pressure fields for the entire a porous medium. The application of the macroscopic method overcomes the difficulties, such as the complexity of the flow in the pores, discontinuity of the flow field, differences of the thermophysical properties of phases, etc. On the other hand, the application of the macroscopic method requires the knowledge of the macroscopic transport properties of the studied porous medium, such as permeability and interfacial heat transfer coefficient [1-3]. The continuity, momentum, and energy equations can be solved for the pore level of a porous medium and then the macroscopic transport parameters, such as permeability, interfacial convective heat transfer coefficient, and thermal dispersion conductivity can be obtained by using volume averaging method $[1,4]$.

There is heat transfer between solid and fluid phases when the local thermal equilibrium between two phases is not valid. Quintard and Whitaker [5,6] discussed the criteria for failing of the local thermal equilibrium in porous media. They suggested constraints that must be satisfied for the validity of the thermal equilibrium assumption. For the local thermal nonequilibrium condition, the heat transfer at the interface of two phases can be modeled by using the interfacial convective heat transfer coefficient. The correlations or diagrams for the determination of the interfacial heat transfer coefficient can be developed experimentally and/or numerically. Recent years, the computational determination of the interfacial convective heat transfer coefficient has been widely employed due to the developments in the numerical

\footnotetext{
${ }^{1}$ Corresponding author.

Contributed by the Heat Transfer Division of ASME for publication in the Journal of Heat TRAnSFER. Manuscript received May 23, 2014; final manuscript received September 25, 2014; published online October 28, 2014. Assoc. Editor: Andrey Kuznetsov.
}

methods and hardware technology. Kuwahara et al. [7] studied the heat and fluid flow between isothermal square rods with staggered arrangement under thermal nonequilibrium condition. A correlation for the Nusselt number was proposed for a wide range of porosity, Prandtl and Reynolds numbers. Nakayama et al. [8] studied anisotropy effect on the heat and fluid flow through an array of square rods in an infinite medium for different macroscopic flow angles and Reynolds numbers. The anisotropy was created by changing the vertical distance between the rods. Saito and de Lemos [9] determined the interfacial heat transfer coefficient numerically for an infinite porous medium in which fully developed flow condition prevails. An array of square rods in staggered arrangement was considered and the results found by Kuwahara et al. [7] were confirmed. Later, Saito and de Lemos [10] determined the interfacial convective heat transfer coefficient for the turbulent flow regime in the same porous medium. They proposed a correlation for the interfacial Nusselt number in terms of porosity, Reynolds and Prandtl numbers for the range of Reynolds numbers greater than 104. Lopez Penha et al. [11] studied fully developed flow through the square rods for both inline and staggered arrangements to determine the macroscopic transport parameters. A constant volumetric heat generation in the rods was considered for the various solid-to-fluid thermal conductivity ratios and Reynolds numbers. Their results indicated that the interfacial Nusselt number is approximately constant for the solidto-fluid thermal conductivity ratios higher than 100 while it varies significantly for lower values. Gamrat et al. [12] performed a study to determine the interfacial Nusselt numbers of banks of square rods in inline and staggered arrangements for low Reynolds number flows $(0.05<\operatorname{Re}<40)$. Both the constant rod temperature and constant volumetric heat generation inside the rods were considered. A relationship between Nusselt number, porosity, Reynolds and Prandtl numbers were shown by the proposed correlations for both arrangements. It was concluded that the thermal boundary condition used for the solid phase affects the heat transfer between the solid and fluid phases in the thermal 
nonequilibrium condition. Alshare et al. [13] focused on the effects of the REV aspect ratio, macroscopic flow direction, and Reynolds number on the interfacial convective heat transfer coefficient for the inline arrangement of square rods. A heat generation in the solid phase was assumed, and it was found that the interfacial heat transfer coefficients are minimal when the flow is along the principal axes of the REV. Further studies on the determination of the interfacial heat transfer coefficient can be found in Refs. [14-16].

As seen from the reported studies, the effects of porosity, Reynolds number, particle arrangement, and flow direction on the interfacial convective heat transfer coefficient were investigated, widely. However, the effects of pore to throat size ratio on the interfacial convective heat transfer coefficient has not been studied. In this study, the interfacial convective heat transfer coefficient of porous media consisting of the rectangular rods with different aspect ratios and having different porosities are computed numerically, and the effects of pore to throat size ratio on the heat transfer coefficient between the solid and fluid are investigated. A correlation for the determination of the interfacial heat transfer coefficient for the wide range of pore to throat size ratio and porosity is developed. The present results might be used specifically for the investigation of heat transfer over pin fin arrays (e.g., Ref. [17]) or slotted fins modeled by porous media approach [18]. It should be mentioned that the geometry of the porous media considered in the present study seems similar to the slotted fin heat sinks. However, there are differences between the present work and classical studies on slotted fin. There is no base plate in this study and the interfacial heat transfer coefficient is defined based on the temperature difference between the fluid in the pores and solid rather than inlet and solid surface. In general, the obtained results provide better understanding of the effect of pore to throat size ratio on the interfacial heat transfer coefficient of any porous medium such as metal foams.

\section{The Considered Porous Media and the Computational Domain}

The schematic of the considered porous media is shown in Fig. 1. The porous media is an infinite media consisting of rectangular rods in inline arrangement. The rectangular rods are assumed very long in the $z$-direction; hence, a two-dimensional model can be used. Considering the periodicity of the porous structure, a REV with the dimensions $H \times H$ is employed to investigate the pore to

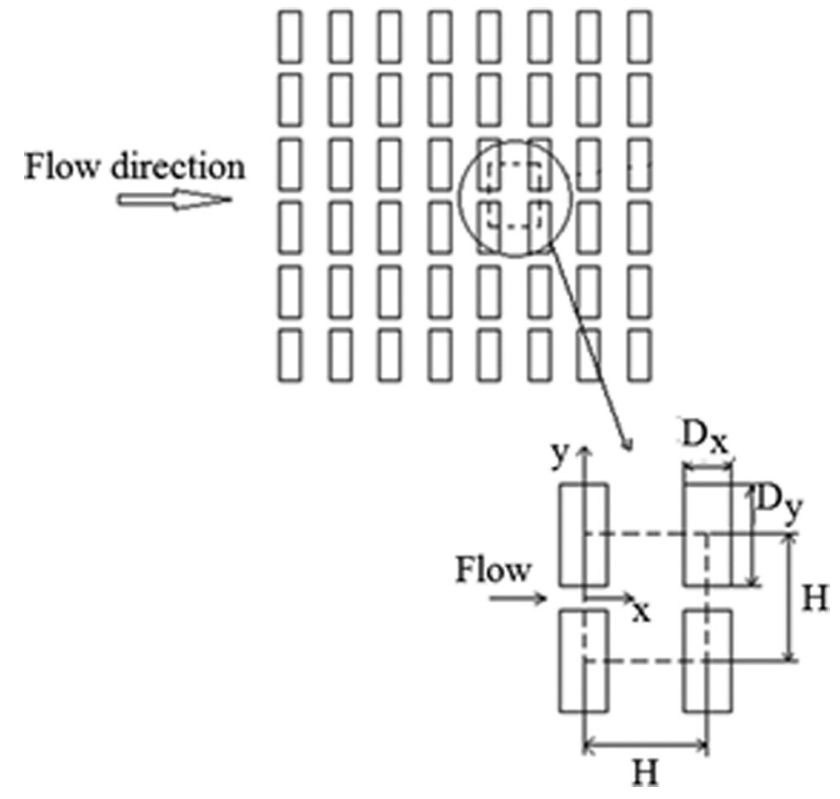

Fig. 1 The schematic view of the considered porous medium throat size ratio effect on convective heat transfer in the porous media. The dimensions of the REV are constant for all studied cases and the rectangular particles have dimensions of $D_{x}$ and $D_{y}$ along $x$ and $y$ directions. The pore to throat size ratio is defined as $\beta=H /\left(H-D_{y}\right)$. The porosity is defined as the ratio of the fluid to total volume of the REV (i.e., $\varepsilon=1-\left(D_{x} D_{y} / H^{2}\right)$ ). The pore to throat size ratio and porosity are changed from 1.63 to 7.46 and from 0.7 to 0.9 , respectively. For instance, Table 1 displays the geometrical parameters of the REV with porosity of 0.7 for different values of $\beta$. The fluid flowing through the porous media is air with constant properties (density of $1.205 \mathrm{~kg} / \mathrm{m}^{3}$ and viscosity of $\left.18.21 \times 10^{-6} \mathrm{~kg} / \mathrm{ms}\right)$. The flow is laminar and macroscopically unidirectional. The Reynolds number (i.e., $\operatorname{Re}=\langle u\rangle H / \nu$ ) is limited between 1 and 100 to avoid of the turbulence and flow instability effects particularly for high pore to throat size ratios. Steady flow with hydrodynamically and thermally periodical and fully developed conditions in the REV is provided, computationally. Furthermore, a uniform temperature distribution is assumed in the solid phase. It should be mentioned that there are huge numbers of porous media with different structures in nature and industry. The present study is limited to a periodic 2D porous media with rectangular long particles to reduce the number of geometrical parameters for much better understanding of the pore to throat size ratio effect on the interfacial heat transfer coefficient.

\section{The Governing Equations and Interfacial Heat Transfer Coefficient}

The fluid flow in the voids between the particles is assumed incompressible and steady. The fluid is a Newtonian fluid with constant thermophysical properties. The steady form of the continuity and momentum equations (Eqs. (1)-(3)) are solved to determine the velocity and pressure fields for the flowing fluid in the voids between the rods. A detailed analysis of the flow configuration and pressure drop in the considered porous media were reported in Ref. [19]. After obtaining the velocity field in the porous media, the energy equation for fluid phase is solved to obtain the temperature distributions in the REV. The energy equation for the solid phase is not taken into account because of the uniform temperature assumption

$$
\begin{gathered}
\frac{\partial u}{\partial x}+\frac{\partial v}{\partial y}=0 \\
u \frac{\partial u}{\partial x}+v \frac{\partial u}{\partial y}=-\frac{1}{\rho} \frac{d p}{d x}+\nu\left(\frac{\partial^{2} u}{\partial x^{2}}+\frac{\partial^{2} u}{\partial y^{2}}\right) \\
u \frac{\partial v}{\partial x}+v \frac{\partial v}{\partial y}=-\frac{1}{\rho} \frac{d p}{d y}+\nu\left(\frac{\partial^{2} v}{\partial x^{2}}+\frac{\partial^{2} v}{\partial y^{2}}\right) \\
u \frac{\partial T_{\mathrm{f}}}{\partial x}+v \frac{\partial T_{\mathrm{f}}}{\partial y}=\frac{1}{\alpha}\left(\frac{\partial^{2} T_{\mathrm{f}}}{\partial x^{2}}+\frac{\partial^{2} T_{\mathrm{f}}}{\partial y^{2}}\right)
\end{gathered}
$$

where $u$ and $v$ are the velocity components along $x$ and $y$ directions, $p$ and $T_{\mathrm{f}}$ represent the pressure and the fluid temperature.

The continuity, momentum, and energy equations, Eqs. (1)-(4), are microscopic equations for the studied porous media. The macroscopic equations for the fluid flow and heat transfer can be

Table 1 A sample for geometrical parameters of the investigated REV

\begin{tabular}{llllll}
\hline \hline & $\beta=1.63$ & $\beta=2.21$ & $\beta=3.04$ & $\beta=4.44$ & $\beta=7.46$ \\
\hline$A^{*}$ & 0.5 & 1 & 1.5 & 2 & 2.5 \\
$\varepsilon$ & 0.7 & 0.7 & 0.7 & 0.7 & 0.7 \\
$D_{x} / H$ & 0.776 & 0.547 & 0.446 & 0.388 & 0.346 \\
$D_{y} / H$ & 0.388 & 0.547 & 0.670 & 0.776 & 0.866 \\
$d_{\mathrm{h}} / H$ & 1.207 & 1.276 & 1.250 & 1.207 & 1.155 \\
$d_{\mathrm{p}} / H$ & 0.776 & 0.820 & 0.804 & 0.776 & 0.742 \\
\hline \hline
\end{tabular}


derived from the microscopic equations by using the volume averaging method $[4,19]$. The volume average of a dependent quantity over the REV can be obtained by Eqs. (5) and (6). Equation (5) shows the total volume average of a quantity $\varphi$ while Eq. (6) is used to find the intrinsic volume averaged value of $\varphi$ for any of the phases in a porous medium

$$
\begin{aligned}
\langle\phi\rangle & =\frac{1}{V} \int_{V} \phi d V \\
\langle\phi\rangle^{x} & =\frac{1}{V_{x}} \int_{V_{x}} \phi d V
\end{aligned}
$$

where $V$ is the total volume of the REV and $V_{x}$ is the volume of the considered phase in the REV ( $s$ or $f$ subscripts refer to the solid or fluid phases, respectively). The variable $\varphi$ can be velocity or temperature in the present study. Taking volume integral of the energy equation (Eq. (4)) and using Eqs. (5) and (6), the following macroscopic energy equation can be found [1]:

$$
\begin{aligned}
\left(\rho c_{\mathrm{p}}\right)_{\mathrm{f}}\left(\langle\vec{u}\rangle \cdot\langle T\rangle^{\mathrm{f}}\right)= & k_{\mathrm{f}} \varepsilon \nabla^{2}\langle T\rangle^{\mathrm{f}}+\nabla\left(\frac{1}{V} \int_{A_{\mathrm{sf}}} k_{\mathrm{f}} T d A\right) \\
& +\frac{1}{V} \int_{\mathrm{A}_{\mathrm{sf}}} k_{\mathrm{f}} \nabla T d A-\left(\rho c_{\mathrm{p}}\right)_{\mathrm{f}} \nabla \cdot\left\langle T^{\prime} \vec{u}^{\prime}\right\rangle
\end{aligned}
$$

The first term on the right hand side of Eq. (7) represents the diffusion heat transfer in the fluid. The second term relates to the thermal tortuosity. The thermal tortuosity term regards the change of the thermal diffusion path due to the different thermal conductivities between the solid and the fluid, and it is described as an elongation in the thermal path due to the existence of the solid particles [20,21]. The last term in Eq. (7) is named as thermal dispersion showing the additional diffusion heat transfer to the molecular diffusion. The third term in the RHS of the macroscopic energy equation shows the heat transfer between the solid phase surface and the fluid flowing in the voids and it can be calculated by using the interfacial convective heat transfer concept. Mathematically, the convective heat transfer between solid and fluid can be expressed by using the interfacial heat transfer coefficient

$$
h_{\mathrm{sf}} A_{\mathrm{ss}}\left(\langle T\rangle^{\mathrm{s}}-\langle T\rangle^{\mathrm{f}}\right)=\frac{1}{V} \int_{A_{\mathrm{sf}}} \vec{n} \cdot k_{\mathrm{f}} \nabla T d A
$$

where $h_{\mathrm{sf}}$ is the interfacial convective heat transfer coefficient, $A_{\mathrm{sf}}$ is the solid-fluid interface area, and $A_{\mathrm{ss}}$ is the specific solid-fluid interface area (i.e., $A_{\mathrm{ss}}=A_{\mathrm{sf}} / V$ ). The interfacial Nusselt number can be defined as follows:

$$
\mathrm{Nu}=\frac{h_{\mathrm{sf}} H}{k_{\mathrm{f}}}
$$

where $H$ represents the height of REV. Although different characteristic lengths such as hydraulic diameter and particle height may be employed to define Nusselt number for the studied porous structure, the dimension of the REV is selected in this study. The same characteristic length is also selected in many reported studies $[8,11,13]$.

Considering the REV in Fig. 1, the boundary conditions for the microscopic equations are chosen as symmetry for the top and the bottom of the REV. A periodic velocity and temperature profiles are generated for the inlet and outlet boundaries. The velocity and temperature gradients at the fluid outlet boundary is assumed zero; hence, no diffusion transport exists. The employed boundary conditions mathematically can be written as follows:

$$
\text { On the solid walls: } u=v=0, \quad T_{\mathrm{f}}=T_{\mathrm{s}}
$$

For the top and bottom boundaries: $\frac{\partial u}{\partial y}=\frac{\partial v}{\partial y}=\frac{\partial T_{\mathrm{f}}}{\partial y}=0$
For the inlet boundary: $u(0, y)=f(y), \quad T(0, y)=g(y)$

$$
\text { For the outlet boundary: } \frac{\partial u}{\partial x}=\frac{\partial v}{\partial x}=\frac{\partial T_{\mathrm{f}}}{\partial x}=0
$$

The functions of $f(y)$ and $g(y)$ are the velocity and temperature profiles, which provide hydraulic and thermal periodicity for the REV. These functions are found by the following method. In order to determine the function of $f(y)$, a velocity profile is assumed at the inlet of the REV. After solving the governing equations for the fluid flow and obtaining the results, the outlet velocity profile is substituted to the inlet boundary. This iterative process continues until the identical velocity profiles at the inlet and outlet boundaries are obtained. Additionally, the value of the permeability for each iterative run is found and the hydraulic periodicity is also checked by the change of permeability value. The value of permeability should not be changed if the hydraulic periodicity for the flow field is achieved. Another iterative process is used to obtain the fully developed heat transfer in the REV. If a thermally fully developed convection heat transfer is valid, no change of the dimensionless temperature and interfacial convective heat transfer should be observed in the sequential REVs through the flow direction in the porous medium. At the beginning of this iterative procedure, a uniform temperature, which is different from the solid temperature, is defined for the fluid inlet boundary and then the temperature field for the entire domain is obtained by solving the energy equation for fluid. The temperature profile at the inlet for the subsequent computation is determined from the dimensionless temperature profile at the outlet boundary of the previous iteration. The iterative process continues until no change in the dimensionless temperature distribution between the inlet and outlet and no variation of the interfacial Nusselt number are observed. The dimensionless temperature is defined by the following equation:

$$
\left.\frac{T-T_{\mathrm{s}}}{T_{\mathrm{b}}-T_{\mathrm{s}}}\right|_{\text {inlet }(x=0)}=\left.\frac{T-T_{\mathrm{s}}}{T_{\mathrm{b}}-T_{\mathrm{s}}}\right|_{\text {outlet }(x=H)}
$$

where $T_{\mathrm{b}}$ is the bulk temperature and it is defined as

$$
T_{\mathrm{b}}=\frac{\int_{-\left(H-D_{y}\right) / 2}^{\left(H-D_{y}\right) / 2} u T d y}{\int_{-\left(H-D_{y}\right) / 2}^{\left(H-D_{y}\right) / 2} u d y}
$$

Furthermore, a local interfacial heat transfer coefficient is defined based on the macroscopic temperature differences between the solid and fluid phases in order to explain the change of the convective heat transfer with $\operatorname{Re}, \varepsilon$, and $\beta$

$$
h_{\mathrm{L}}\left(\langle T\rangle^{\mathrm{s}}-\langle T\rangle^{\mathrm{f}}\right)=\vec{n} \cdot k_{\mathrm{f}} \nabla T
$$

where $\nabla T$ is the local temperature gradient through the solid-fluid interface. Hence, the local interfacial Nusselt number (relates to the Nusselt number at any point of the solid-fluid interface), $\mathrm{Nu}_{\mathrm{L}}$, is defined as

$$
\mathrm{Nu}_{\mathrm{L}}=\frac{h_{\mathrm{L}} H}{k_{\mathrm{f}}}
$$

The interfacial Nusselt number can be calculated directly from Eqs. (8) and (9), or by the determination of area based average of the local interfacial Nusselt number, calculated from Eq. (17).

\section{The Numerical Procedure and the Computational Details}

The microscopic flow and energy equations are solved for the considered REVs, computationally. The grid size is $500 \times 250$ for 


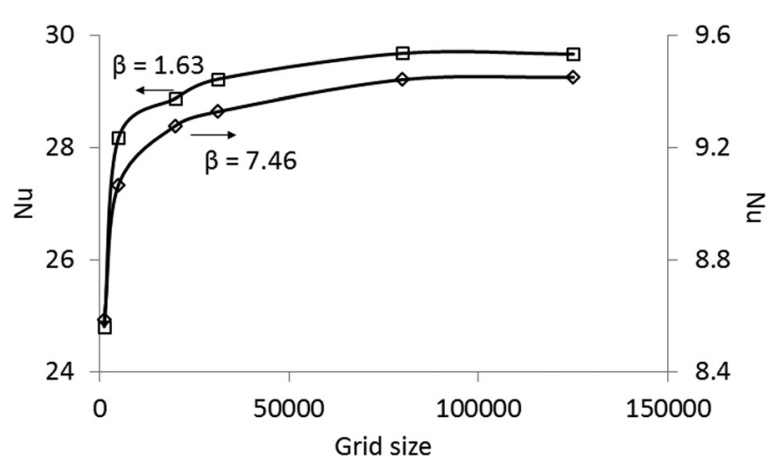

Fig. 2 The effect of number of grid on the obtained Nusselt number for $\varepsilon=0.9, \operatorname{Re}=100$, and $\beta=1.63$ and $\beta=7.46$

half of the REV, which is chosen as the computational domain. A commercial code (ANSYS 12/FLUENT) based on the finite volume method is used to solve the governing equations. The power law scheme is employed for the discretization of the convection terms in the momentum and energy equations. Semi-implicit method for pressure linked equations (SIMPLE) method is used for handling the pressure-velocity coupling [22]. The approximate errors are set to $10^{-9}$ for flow equations and $10^{-12}$ for temperature field. Grid independency study is done for two extreme cases as $\beta=1.63$ and $\beta=7.46$ when $\varepsilon=0.9$ and $\operatorname{Re}=100$, and the results are displayed in Fig. 2. As can be seen, the employed grid number is sufficient to discretize the computational domain to achieve an accurate value for the interfacial Nusselt number.

\section{Results and Discussion}

For the validation of the present numerical results, computations are performed for a periodic porous medium constructed with square rods in inline arrangement with the porosity of 0.75 . The obtained interfacial Nusselt number for this porous medium is compared with the results of Nakayama et al. [8], Lopez Penha et al. [11], and Gamrat et al. [12] and displayed in Fig. 3. As seen, there is good agreement between the results of the present and reported studies, which validates the obtained results of present study.

The change of velocity profiles at the inlet and outlet of the REV to achieve a periodic flow in the porous medium with $\beta=1.63, \varepsilon=0.7$, and $\operatorname{Re}=100$ are shown in Fig. 4. At the beginning of the numerical computation, a parabolic velocity profile for the inlet of the REV (the inlet of first run) is determined and assigned to the program. To obtain a periodic velocity distribution, an iterative process is performed by substituting the computed outlet velocity profile into the inlet of the REV and performing further computation until the inlet and outlet velocity profiles become identical (e.g., the inlet and outlet of the third run). Similarly, the periodicity of the temperature field is succeeded by applying an iterative process. The change of the dimensionless temperature profiles at the inlet and outlet of the REV through the iterative procedure for obtaining a periodical

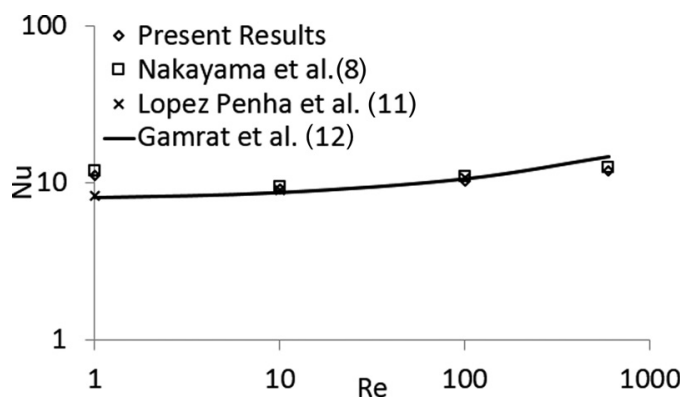

Fig. 3 The comparison of the present numerical results with reported studies in literature

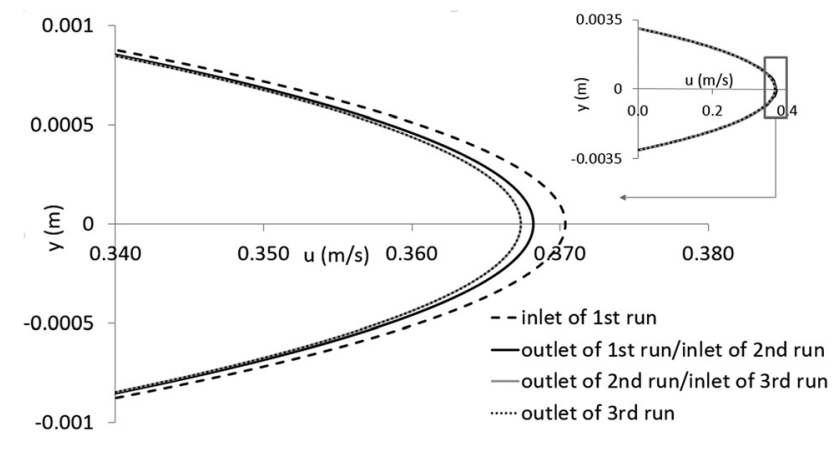

Fig. 4 The change of velocity profiles at the inlet and outlet through the iterative procedure to obtain periodic velocity distribution in the studied $\operatorname{REV}(\beta=1.63, \varepsilon=0.7$, and $\operatorname{Re}=100)$

temperature field is shown in Fig. 5 for the same porous medium. At the beginning of the iterative procedure, a uniform temperature profile for the inlet of the REV (i.e., the inlet of first run) is assigned to the program. Then, the dimensionless temperature profile at the outlet of the REV is calculated based on the computed outlet temperature profile. The inlet temperature profile is calculated from the dimensionless outlet temperature profile according to Eq. (14) and the computation is repeated. The iterative procedure is terminated when the dimensionless temperature profiles at the inlet and outlet of the REV become identical and the change of the interfacial Nusselt number becomes negligible (e.g., the inlet and outlet temperature profile of the fifth run).

The Effects of Pore to Throat Size Ratio on Interfacial Nusselt Number. The streamlines and temperature contours for $\beta=1.63, \varepsilon=0.7$ and for different Re numbers are displayed in Fig. 6. In order to compare different temperature fields, a dimensionless temperature as $\theta_{\mathrm{f}}=\left(T_{\mathrm{f}}-T_{\min }\right) /\left(T_{\max }-T_{\min }\right)$ is defined, where $T_{\min }$ and $T_{\max }$ are the minimum and maximum temperatures in the REV, respectively. For all presented Reynolds numbers, two types of flow as main and secondary flows are observed. The main flow generally resembles a clear channel flow through the porous medium while the secondary flows occur in the gaps between the particles. The temperature distribution considerably changes with $\operatorname{Re}$ number. For $\operatorname{Re}=1$, the fluid slowly passes through the REV (i.e., weak convective transport) and its residence time in the REV is longer. As a result, the fluid temperature can increase to the solid temperature just after the inlet throat and the volume averaged dimensionless fluid temperature is high $\left(\left\langle\theta_{\mathrm{f}}\right\rangle^{\mathrm{f}}=\left(\langle T\rangle^{\mathrm{f}}-T_{\min }\right) /\left(T_{\max }-T_{\min }\right)=0.862\right)$. The local heat transfer coefficient near the inlet section is expected to be very large compared to other surfaces of the solid in REV. As Reynolds number increases to $\mathrm{Re}=10$, the convective heat transport

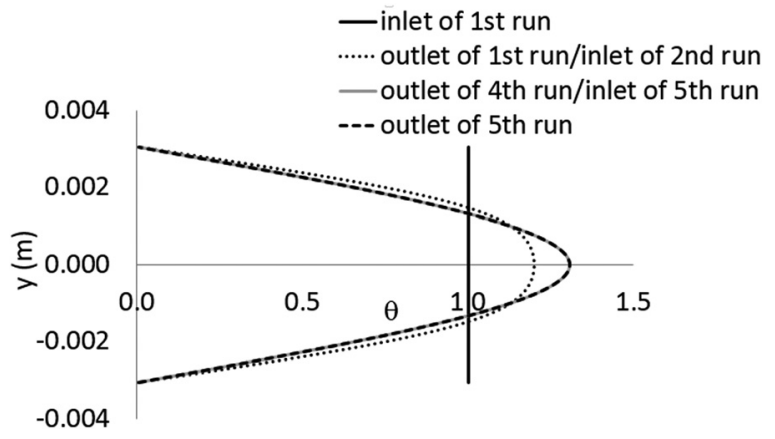

Fig. 5 The change of dimensionless temperature $(\theta)$ profiles at the inlet and outlet through the iterative procedure to obtain periodic temperature distribution in the studied REV $(\beta=1.63$, $\varepsilon=0.7$, and $R e=100$ ) 


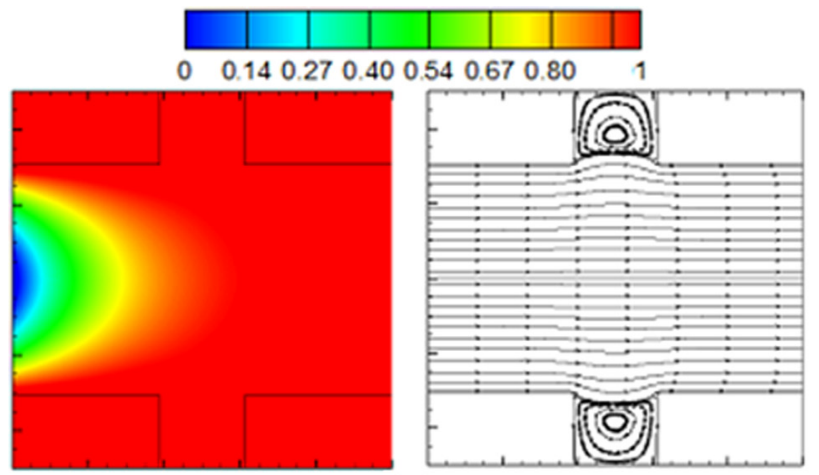

(a)
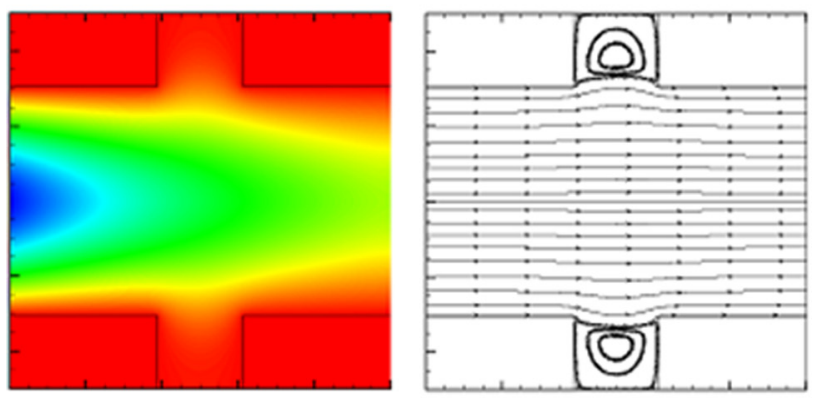

(b)
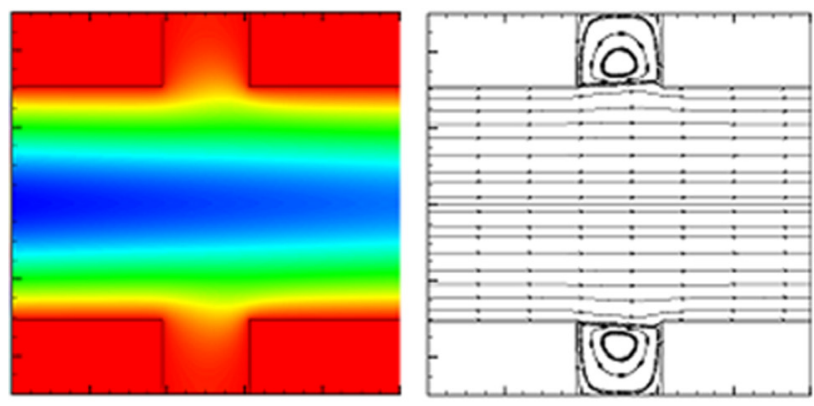

(c)

Fig. 6 The streamlines (on the right) and temperature contours (on the left) for $\beta=1.63$ and $\varepsilon=0.7$, (a) $\operatorname{Re}=1,(b) \operatorname{Re}=10$, and (c) $\operatorname{Re}=100$

becomes stronger and the residence time of the fluid particles in the porous medium diminishes. Furthermore, thermal boundary layers occur on the horizontal surfaces of the solid particles, which create a thermal resistance for the heat flow in the transverse direction of the fluid flow. As a result, a remarkable temperature difference between the center and the solid surface region is observed. The volume averaged dimensionless temperature, $\left\langle\theta_{\mathrm{f}}\right\rangle^{\mathrm{f}}$, reduces to 0.673. Further increase in $\operatorname{Re}$ number $(\operatorname{Re}=100)$ causes the decrease of the thermal boundary layer thickness. As Re increases, the convection heat transfer become stronger, the residence time of the fluid in the REV decreases, and a uniform temperature along the flow direction is observed. For $\mathrm{Re}=100$, $\left\langle\theta_{\mathrm{f}}\right\rangle^{\mathrm{f}}$ becomes as 0.472 .

The variation of local interfacial Nusselt numbers along the solid-fluid interface for $\beta=1.63$ are displayed in Fig. 7. The figure indicates that the local interfacial Nusselt number value for $\mathrm{Re}=1$ is the highest at the inlet of the REV (i.e., point a) and then a sharp decrease is seen toward the edge of the inlet particles (i.e., point b). The reason of the high value of $\mathrm{Nu}_{\mathrm{L}}$ at the inlet is the long residence time of the fluid in the inlet region due to the low velocity. The convective transport becomes stronger by increase of Re and consequently the residence time of the fluid and the value of $\mathrm{Nu}_{\mathrm{L}}$ in the inlet region decrease. The stronger convection transport causes the

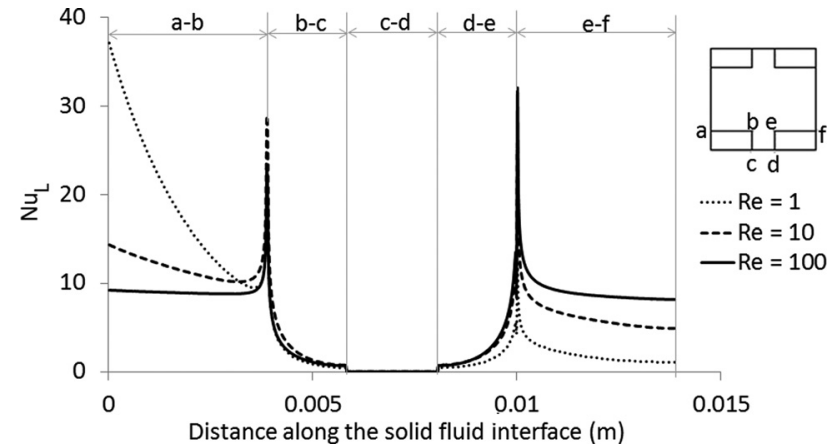

Fig. 7 The variations of local interfacial Nusselt number along the solid-fluid interface for $\beta=1.63$ and $\varepsilon=0.7$

increase of $\mathrm{Nu}_{\mathrm{L}}$ on the interface of the right particles in the REV (d-e and e-f surfaces) since the solid surface touches colder fluid. For $\mathrm{Re}=100$, the value of $\mathrm{Nu}_{\mathrm{L}}$ are almost constant at the inlet and outlet throat of the REV ( $a-b$ and $e-f$ surfaces). Although $\mathrm{Nu}_{\mathrm{L}}$ values at the interfaces considerably vary with Re and the location, one may find that the average interfacial Nusselt number (i.e., the area between the curves and $x$-axis in Fig. 7) may be close to each other.

For $\beta=7.46$, the corresponding streamlines and the dimensionless temperature contours are displayed in Fig. 8. As the value of $\beta$ increases, the vertical distance between the solid particles decreases and the throat effect becomes stronger. Similar to the flows presented in Fig. 6, there are secondary flows in the top and bottom gaps between the particles. For $\operatorname{Re}=1$, the main flow penetrates into the gaps and distorts the secondary flows in such way that two additional vortices occur in the main secondary flows. The temperature distribution is mainly influenced by the conduction heat transfer and the fluid temperature rapidly increases to the solid temperature when the main flow passes through the inlet throat. The residence time of the fluid in the porous structure is long and $\left\langle\theta_{\mathrm{f}}\right\rangle^{\mathrm{f}}$ is very close to the dimensionless solid temperature $\left(\left\langle\theta_{\mathrm{f}}\right\rangle^{\mathrm{f}}=0.991\right)$. By the increase of Re to 10 , the main flow enters into the gaps and the fluid particles crush the vertical walls of the right solid particles of the REV. The thermal boundary layer on the horizontal sides of the inlet solid particles can be seen from the dimensionless temperature contour of $\mathrm{Re}=10$. The influence of fluid inlet temperature is enhanced as Re increases. The value of $\left\langle\theta_{\mathrm{f}}\right\rangle^{\mathrm{f}}$ decreases to 0.891 for $\mathrm{Re}=10$. As Re increases to 100 , the residence time in the REV further decreases. For this Re, the secondary flows play an important role on the temperature distribution in the REV. A strong crush of the fluid with the vertical edges of the outlet solid particles causes the penetration of the heat into the gaps. Due to the strong convective heat transport, the fluid temperature remains smaller than the solid temperature and $\left\langle\theta_{\mathrm{f}}\right\rangle^{\mathrm{f}}=0.73$.

The variations of $\mathrm{Nu}_{\mathrm{L}}$ along the solid-fluid interface for different Re are displayed in Fig. 9 when $\beta=7.46$. For $\operatorname{Re}=1$, the value of $\mathrm{Nu}_{\mathrm{L}}$ in the inlet section of the left is considerably greater than the outlet section. This change of $\mathrm{Nu}_{\mathrm{L}}$ is expected since the temperature gradient in the inlet of the REV is much higher than the remaining part. For $\operatorname{Re}=10$ and 100, the heat transfer occurs both in the inlet and outlet throats and on the vertical sides of the solid particles (especially at of the right particles). The heat transfer on the surfaces of the inlet particles for $\mathrm{Re}=10$ considerably decreases while an increase of the heat transfer in the outlet particle of the REV is observed. For $\operatorname{Re}=100$, the considerable increase of the heat transfer on the vertical walls of the right particles is due to the strong crush of fluid to the vertical walls. An increase in the interfacial Nusselt number for $\mathrm{Re}=100$ may be expected.

The variations of the interfacial $\mathrm{Nu}$ with Reynolds number for the studied pore to throat size ratio values are shown in Fig. 10 for $\varepsilon=0.7$. Additionally, the interfacial $\mathrm{Nu}$ for the flow in a straight channel with thick walls when $\varepsilon=0.7$ is shown on the same figure. The lowest interfacial $\mathrm{Nu}$ are obtained for the straight channel flow and it does not change with Re, since the flow is thermally 


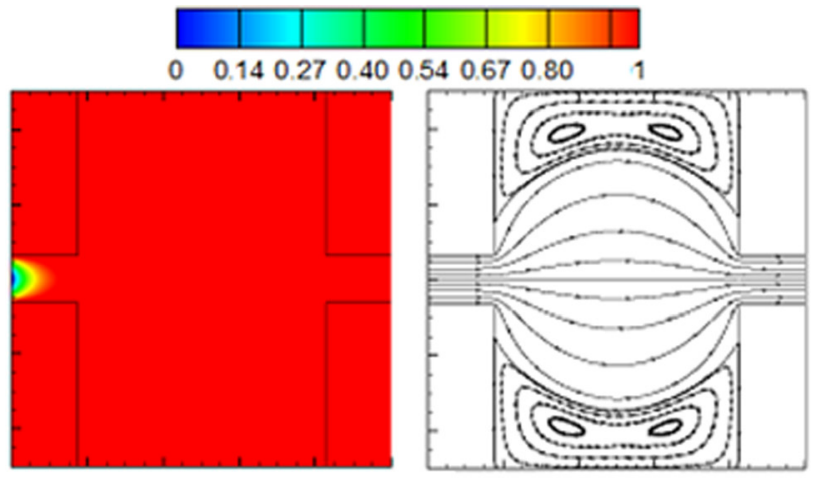

(a)
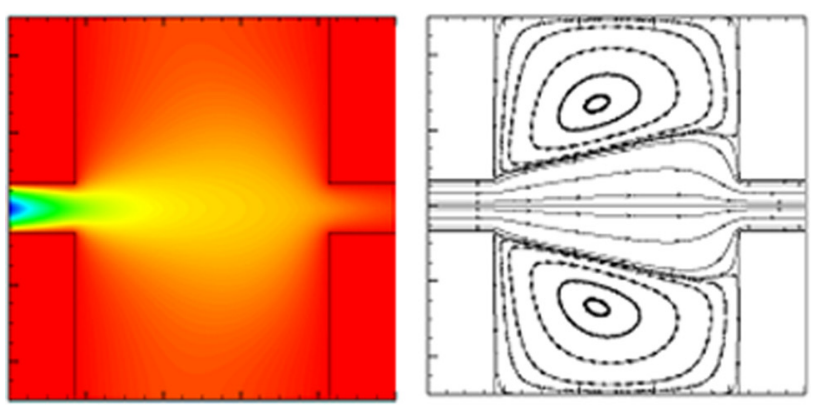

(b)
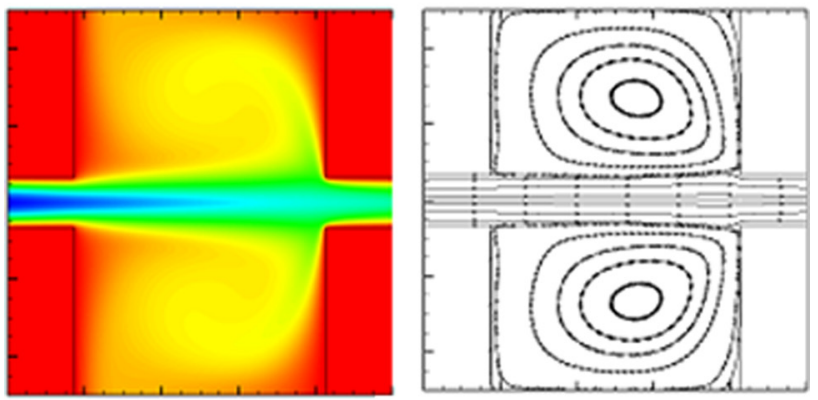

(c)

Fig. 8 The streamlines (on the right) and temperature contours (on the left) for $\beta=7.46$ and $\varepsilon=0.7$, (a) $R e=1$, (b) $R e=10$, and (c) $\mathrm{Re}=100$

fully developed. For porous structures with $\varepsilon=0.7$, the increase of interfacial $\mathrm{Nu}$ with $\beta$ is observed. The increase of $\beta$ causes the mixing of the fluid in the voids between the particles. For $\mathrm{Re}=1$ and $\beta=1.63$, the value of $\left\langle\theta_{\mathrm{f}}\right\rangle^{\mathrm{f}}=0.862$ while it is 0.991 for $\beta=7.46$ due to the mixing effect. For $\beta=1.63$ and 2.21 , a small

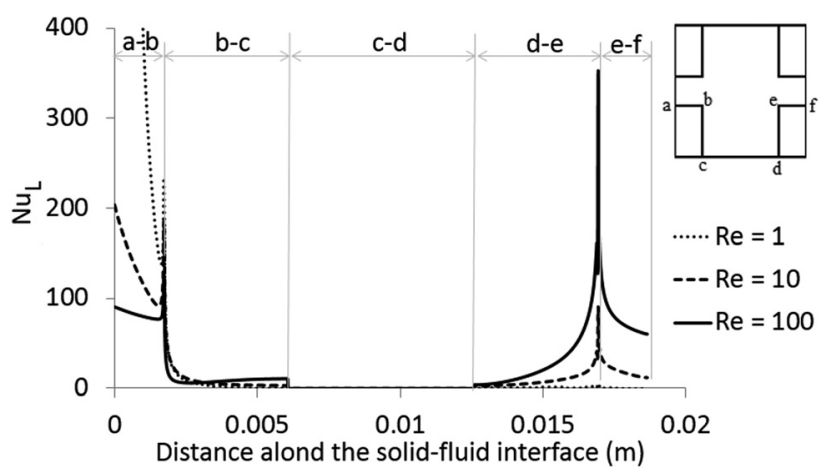

Fig. 9 The variations of local interfacial Nusselt numbers along the solid-fluid interface for $\beta=7.46$ and $\varepsilon=0.7$

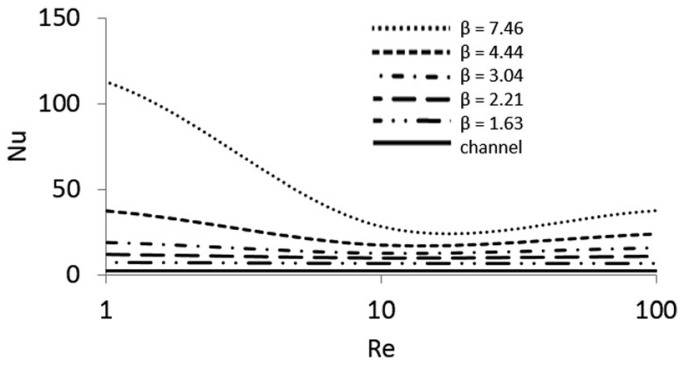

Fig. 10 The change of interfacial Nusselt number with Reynolds number for porous structure with $\varepsilon=0.7$ and different pore to throat size ratios

change of the interfacial $\mathrm{Nu}$ with Re is observed since the flow is similar to the channel flow. Further increase of $\beta$ causes a dramatic change of the interfacial $\mathrm{Nu}$ with $\mathrm{Re}$. For high $\beta$ values (e.g., $\beta=7.46$ ), the interfacial $\mathrm{Nu}$ decreases along the region of Re between 1 and 10 and takes a minimum value at $R e=10$. The decrease of $\mathrm{Nu}$ may be due to the decrease of the residence time of the fluid in the porous media and the formation of the thermal boundary layer on the horizontal surfaces of the inlet solid particles cause the decline of $\mathrm{Nu}_{\mathrm{L}}$ in the inlet region. Further increase of Re from 10 to 100 increases the interfacial $\mathrm{Nu}$. Based on our observation, the reason of the increase of $\mathrm{Nu}$ with $\mathrm{Re}$ for high values of $\beta$ for the region of $\operatorname{Re}>10$ is the crush of the fluid to the vertical wall of the outlet particle, as indicated before. The increase of the $\mathrm{Nu}_{\mathrm{L}}$ at the vertical walls of the right particle for $\mathrm{Re}=100$ can be observed from Fig. 8 .

In Table 1 , the geometrical properties of the studied REVs representing periodic porous media with $\varepsilon=0.7$ are given. The pore hydraulic and equivalent particle diameters of REVs with $\beta=1.63$ and $\beta=4.44$ are the same as well as their porosity. However, as can be seen from Fig. 10, the values of interfacial $\mathrm{Nu}$ of these two porous media are considerably different for the same Re. Hence, Nu cannot be determined only based on the geometrical parameters such as $d_{\mathrm{h}}, d_{\mathrm{p}}$, and $\varepsilon$, and the pore to throat size ratio should be taken into consideration in order to develop a general correlation for the determination of interfacial Nusselt number for a porous medium.

The Effect of Porosity on Interfacial Nusselt Number. The change of the local interfacial Nusselt number for $\beta=1.63$ and $\mathrm{Re}=1$ for the porosities from 0.7 to 0.9 is shown in Fig. 11(a). As can be seen, the most of the heat transfer between the surfaces of solid particles and the fluid occurs at the inlet throat of the REVs. For low Re, the horizontal surfaces of the particles play an important role on the heat transfer compared to the vertical walls. The comparison of the change of $\mathrm{Nu}_{\mathrm{L}}$ for $\varepsilon=0.7$ and 0.9 shows that the interfacial $\mathrm{Nu}$ may not be higher for the porous medium with porosity of 0.7 due to longer surface for $\varepsilon=0.7$.

The change of $\mathrm{Nu}_{\mathrm{L}}$ for $\beta=1.63$ and $\mathrm{Re}=100$ for the studied porosities are displayed in Fig. 11(b). The values of $\mathrm{Nu}_{\mathrm{L}}$ of the inlet and outlet regions are comparable and almost have a symmetrical distribution for $\varepsilon=0.7$. The values of $\mathrm{Nu}_{\mathrm{L}}$ increases sharply near the outlet region of the porous medium due to the strike of the fluid to the vertical walls of the right particles in the REVs.

The variation of the interfacial $\mathrm{Nu}$ with $\mathrm{Re}$ for $\beta=1.63$ and for different porosities are shown in Fig. 12. The interfacial $\mathrm{Nu}$ values change between 7 and 10 for the studied porosities when $\beta=1.63$. The interfacial $\mathrm{Nu}$ increases with $\varepsilon$ due to the increase of the void for proper mixing of the fluid. For porosity of 0.7 , the interfacial $\mathrm{Nu}$ is almost constant and similar to the fully developed flow in the channel. For higher porosities, $\mathrm{Nu}$ decreases with the increase of Re due to the occurrence of the boundary layer on the horizontal surfaces of the solid particles and the decrease of the residence time of the fluid in the REV. However, for $\varepsilon=0.8$ and 0.9 , an increase of $\mathrm{Nu}$ is observed after $\mathrm{Re}=10$. The expansion of the 


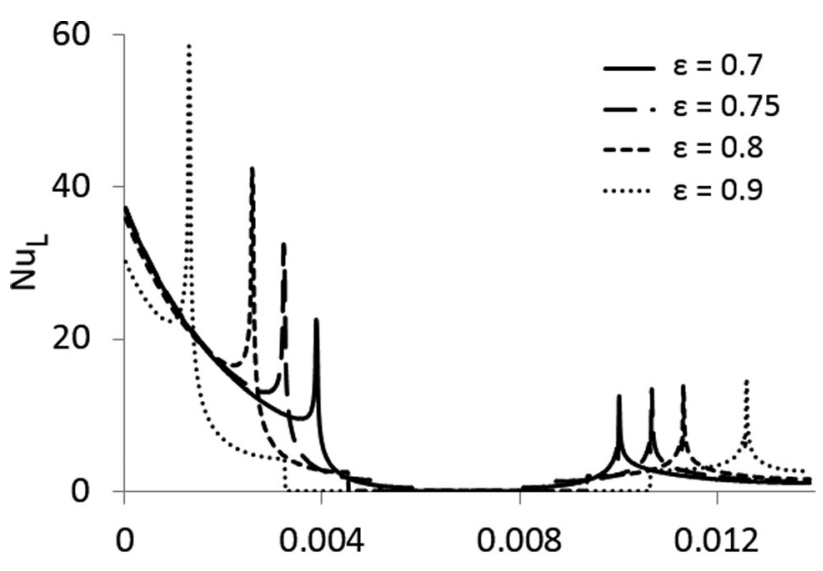

Distance along the solid fluid interface $(\mathrm{m})$

(a)

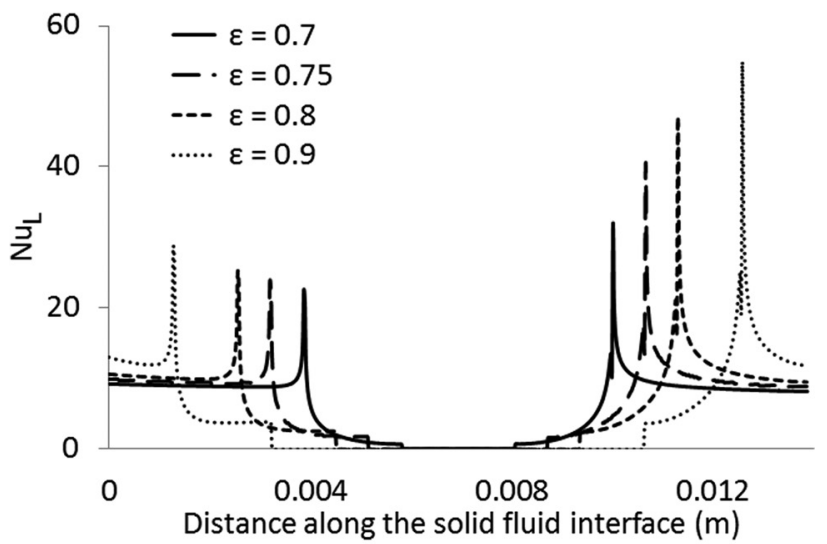

(b)

Fig. 11 The variations of local interfacial Nusselt numbers along the solid-fluid interfaces with porosity for $\beta=1.63$ (a) $\operatorname{Re}=1$ and $(b) \operatorname{Re}=100$

flow after leaving the inlet throat and then the strike of the fluid to the vertical walls of the outlet solid particles in the REV causes the mixing of the fluid in the REV. The effect of the strike of the fluid to the vertical walls of the outlet particles in the REV can be observed by the comparison of the isotherms for $\varepsilon=0.7$ and 0.9 when $\beta=1.63$ and $\operatorname{Re}=100$ (Fig. 13). As can be seen, the strike of the flowing fluid to the vertical walls of the outlet solid particles causes the entrance of the colder fluid into the top and bottom gaps between the particles. As a result, the heat transfer on the vertical walls of the particles increases.

The change of $\mathrm{Nu}_{\mathrm{L}}$ along the solid-fluid interface for $\beta=7.46$, $\operatorname{Re}=1$, and different porosities are displayed in Fig. 14(a). For the flows with low Re, the heat is mainly transferred from the horizontal surfaces of the inlet solid particles of the REV. Hence, the

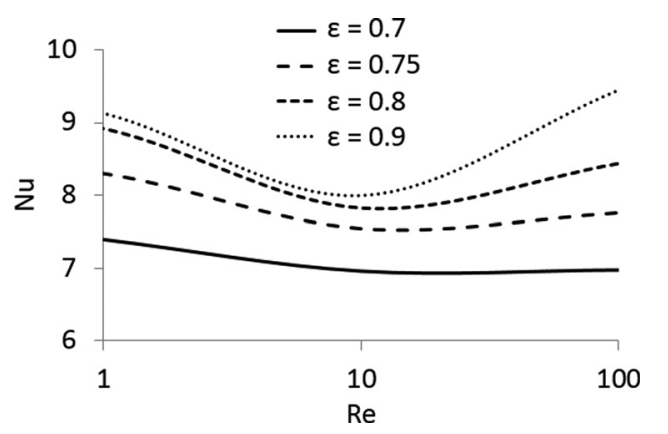

Fig. 12 The change of interfacial Nusselt number with Re number for $\beta=1.63$ and different porosities

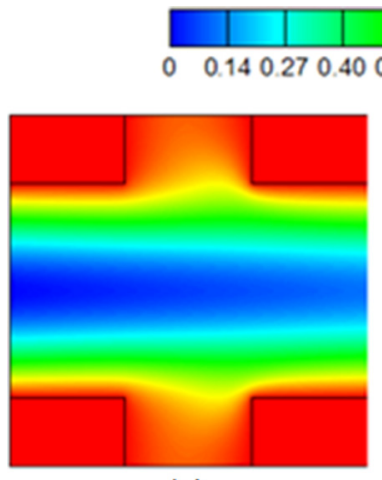

(a)

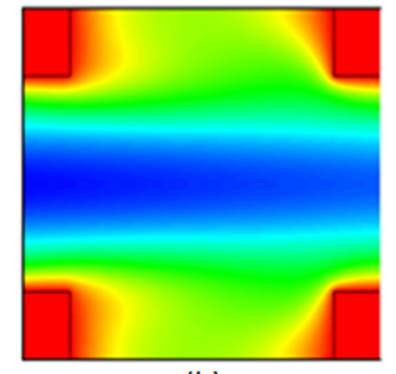

(b)
Fig. 13 The dimensionless temperature contours in porous media with $\operatorname{Re}=100$ and $\beta=1.63$, (a) $\varepsilon=0.75$ and $(b) \varepsilon=0.9$

heat transfer mainly occurs at the inlet throat and it is negligible at the rest of the REV. The value of $\mathrm{Nu}_{\mathrm{L}}$ decreases as $\varepsilon$ increases due to the decrease of the horizontal surface along the throat length. The change of $\mathrm{Nu}_{\mathrm{L}}$ for $\beta=7.46$, the porosity from 0.7 to 0.9 and $\mathrm{Re}=100$ is shown in Fig. 14(b). The changes of $\mathrm{Nu}_{\mathrm{L}}$ for all porosities are similar to each other. The strong crush of the fluid to the vertical walls of the right solid particles in the REV and the strong secondary flows in the gap between the particles cause the increase of $\mathrm{Nu}_{\mathrm{L}}$ at the vertical surfaces of the particle in REV.

The change of the interfacial $\mathrm{Nu}$ with $\mathrm{Re}$ for $\beta=7.46$ is shown in Fig. 15. The values of $\mathrm{Nu}$ becomes closer to each other as $\mathrm{Re}$ increases and they are almost the same at $\mathrm{Re}=100$. This behavior can be explained by Fig. $14(b)$ where the areas under $\mathrm{Nu}_{\mathrm{L}}$ curves are comparable with each other and additionally, the length of the solid particles are almost close to each other. It is observed that

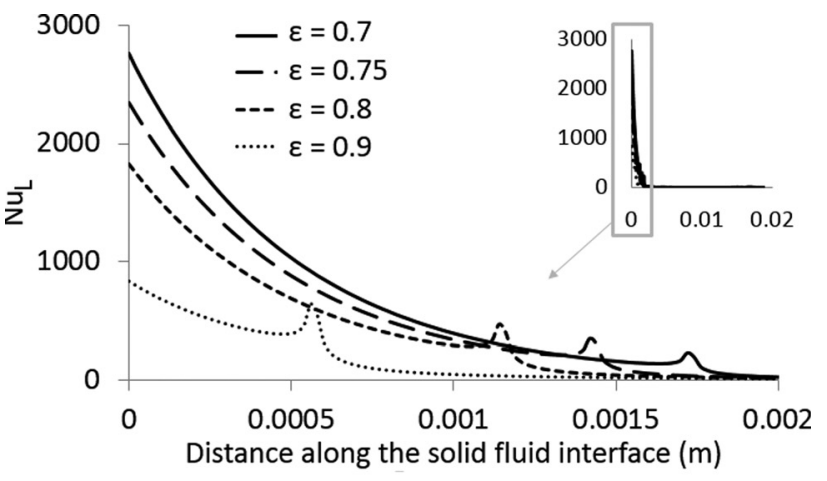

(a)

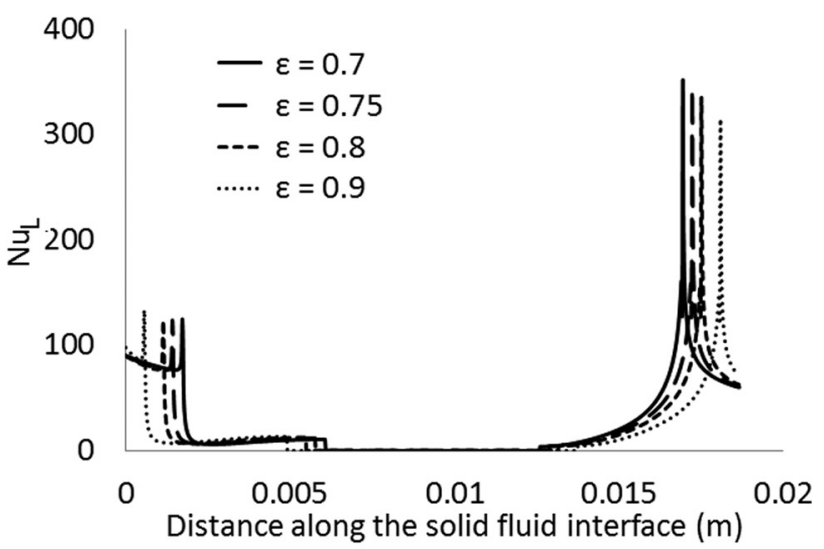

(b)

Fig. 14 The variations of local interfacial Nusselt numbers along the solid-fluid interfaces with porosity for $\beta=7.46$, (a) $\operatorname{Re}=1$ and $(b) \operatorname{Re}=100$ 


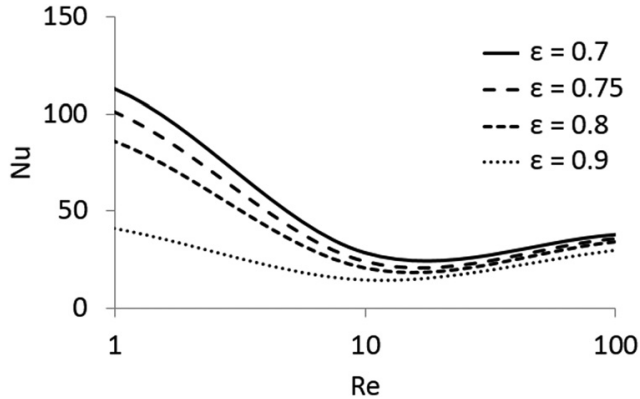

Fig. 15 The change of interfacial Nusselt number with Re number for porous media with $\beta=7.46$

for high $\beta$ values (i.e., $\beta=7.46$ ), the increase of $\varepsilon$ reduces $\mathrm{Nu}$ and this behavior is the reverse of the change of $\mathrm{Nu}$ with $\varepsilon$ for the low $\beta$ values (e.g., $\beta=1.63$ ).

In the light of the present numerical results, a correlation relating the interfacial Nusselt number with porosity, pore to throat size ratio, and Reynolds and Prandtl numbers is developed for the considered porous media. The proposed correlation is shown in the following equation:

$$
\mathrm{Nu}=\left(a_{0} \varepsilon+a_{1}\right)+\left(a_{2} \varepsilon+a_{3}\right) \operatorname{Re}^{0.6} \operatorname{Pr}^{1 / 3}
$$

where $a_{i}$ are functions of $\beta$ and defined as

$$
a_{i}=a_{i 1}+a_{i 2} \beta+a_{i 3} \beta^{2}
$$

Table 2 The empirical coefficients of the proposed correlation for interfacial Nusselt number

\begin{tabular}{lcc}
\hline \hline & $\operatorname{Re}<10$ & $\operatorname{Re}>10$ \\
\hline$a_{01}$ & -12.164 & 20.96 \\
$a_{02}$ & 30.362 & -13.555 \\
$a_{03}$ & -11.581 & 0.0149 \\
$b_{01}$ & 19.699 & -10.926 \\
$b_{02}$ & -28.234 & 11.771 \\
$b_{03}$ & 11.551 & 0.0561 \\
$c_{01}$ & 8.5755 & 0.5923 \\
$c_{02}$ & -10.652 & 0.1241 \\
$c_{03}$ & 2.879 & 0.0172 \\
$d_{01}$ & -8.3585 & -0.932 \\
$d_{02}$ & 10.097 & 0.2443 \\
$d_{03}$ & -2.8843 & -0.032 \\
\hline \hline
\end{tabular}

The values of the empirical coefficients are given in Table 2. Our observation reveals that the variation of $\mathrm{Nu}$ with $\mathrm{Re}$ is considerably different for the regions before and after $\mathrm{Re}=10$. Hence, the coefficients are found for two different regions of Re as can be seen in Table 2. The values of the interfacial $\mathrm{Nu}$ found from the computational study and the suggested correlation are compared in Fig. 16(a). As can be seen, the proposed correlation provides acceptable values for the interfacial $\mathrm{Nu}$ of a porous medium consists of rectangular rods.

Additionally, the comparison between the proposed correlation and the results reported in the literature for square rods in inline
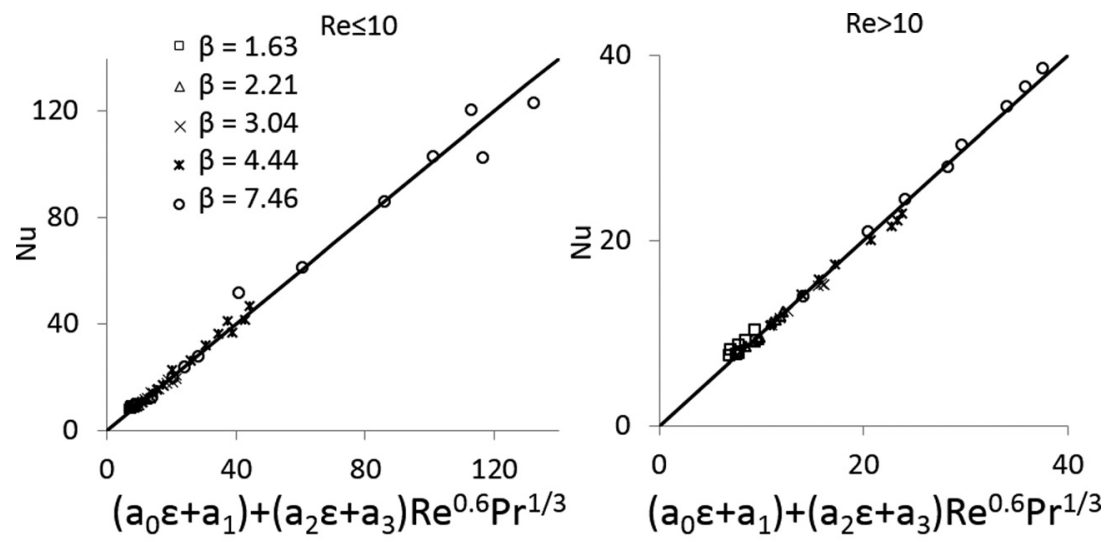

(a)

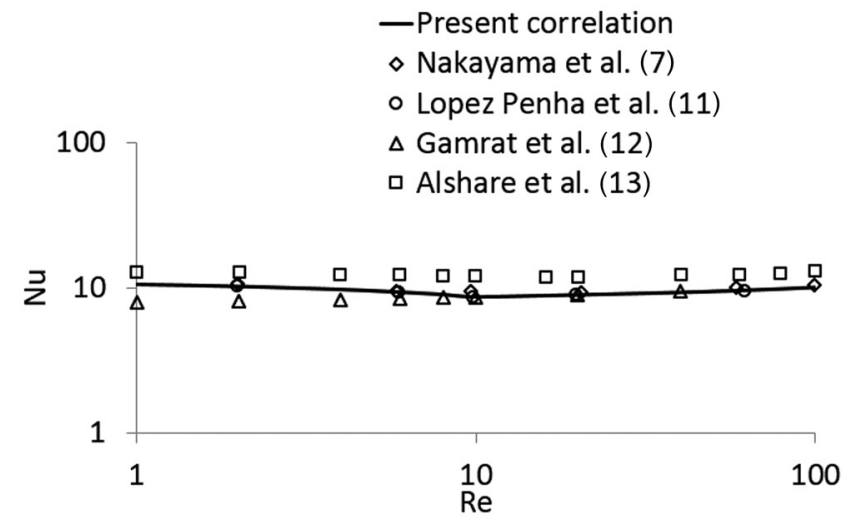

(b)

Fig. 16 The applicability of the correlation (a) the comparison of the obtained numerical values of Nu values with the suggested correlation, $(b)$ the comparison of the suggested correlation with the reported values in literature for $\varepsilon=0.75$ and $\beta=2$

012602-8 / Vol. 137, JANUARY 2015

Transactions of the ASME 
arrangement $(\varepsilon=0.75$ and $\beta=2)$ is shown in Fig. $16(b)$. The Nusselt numbers found by the present correlation seem to align with the results achieved by other researchers.

\section{Conclusion}

The effects of pore to throat size ratio on the interfacial convective heat transfer coefficient are investigated numerically for the porous media consisting of rectangular rods. The present study reveals that the pore to throat size ratio is an important parameter, which should be taken into account for the determination of the convective heat transfer coefficient. A correlation in terms of porosity, pore to throat size ratio, and Reynolds and Prandtl numbers is suggested for the determination of the interfacial Nusselt number in porous media with rectangular rods. Based on the obtained results and the performed discussion, following remarks can be concluded for a periodic two dimensional porous media with long rectangular rods:

- The interfacial convective heat transfer coefficients of two porous media having the same porosity, equivalent particle and pore hydraulic diameters can be considerably different. The pore to throat size ratio is a significant parameter, which can considerably influence the interfacial convective heat transfer coefficient.

- The increase of pore to throat size ratio causes the mixing of the fluid in the voids between the particles and consequently the interfacial Nusselt number increases.

- For the porous media with the low value of pore to throat size ratio (i.e., $\beta=1.63$ ), the interfacial Nusselt number is almost constant, and the heat transfer in the porous media behaves similar to the fully developed heat transfer in a straight channel.

- For the porous media with high values of pore to throat size ratio (e.g., $\beta=7.46$ ), the interfacial heat transfer coefficient decreases for the range up to $\operatorname{Re}=10$ and then it increases with Re. The strike of the fluid to the vertical walls of the outlet solid particles and the entrance of fluid into the gaps between the particles are the main reasons for the enhancement of heat transfer for the region of $\operatorname{Re}>10$.

- Two opposite behaviors are observed for the effect of porosity on the interfacial Nusselt number. For the low pore to throat size ratios, the increase of porosity enhances the Nusselt number because the flow is penetrated deeply into the gaps and the vertical walls of solid particle contribute on the heat transfer. For high pore to throat size ratios, the Nusselt number decreases with the increase of porosity due to the reduction of horizontal surface of solid particles.

\section{Acknowledgment}

The authors acknowledge the Scientific and Technical Research Council of Turkey, TUBITAK, for Ph.D. student scholarship. This study was also supported by Izmir Institute of Technology with Scientific Research Project number of 2012-IYTE-02.

\section{Nomenclature}

$A_{\text {sf }}=$ interfacial area between solid and fluid phases $\left(\mathrm{m}^{2}\right)$ $A_{\mathrm{ss}}=$ specific solid-fluid interface area $\left(\mathrm{m}^{2} / \mathrm{m}^{3}\right)$

$A^{*}=$ aspect ratio of rods $\left(=D_{y} / D_{x}\right)$

$c_{\mathrm{p}}=$ specific heat of fluid $(\mathrm{J} / \mathrm{kgK})$

$D_{x}=$ dimension of solid particle along $x$-direction (m)

$D_{y}=$ dimension of solid particle along $y$-direction $(\mathrm{m})$

$d_{\mathrm{h}}=$ pore hydraulic diameter $(\mathrm{m})\left(=4 \varepsilon /\left[A_{0}(1-\varepsilon)\right]\right)$

$d_{\mathrm{p}}=$ equivalent particle diameter $(\mathrm{m})$

$\stackrel{H}{H}=$ dimension of structural unit $(\mathrm{m})$

$h_{\mathrm{L}}=$ local convective heat transfer coefficient $\left(\mathrm{W} / \mathrm{m}^{2} \mathrm{~K}\right)$

$h_{\mathrm{sf}}=$ interfacial convective heat transfer coefficient $\left(\mathrm{W} / \mathrm{m}^{2} \mathrm{~K}\right)$ $k_{\mathrm{f}}=$ thermal conductivity of fluid $(\mathrm{W} / \mathrm{mK})$

$\mathrm{Nu}=$ interfacial Nusselt number

$\mathrm{Nu}_{\mathrm{L}}=$ local interfacial Nusselt number

$p=$ pressure $(\mathrm{Pa})$

$\operatorname{Pr}=$ Prandtl number

$\mathrm{Re}=$ Reynolds number $(=\rho \mathrm{u} H / \mu)$

$T=$ temperature $(\mathrm{K})$

$u=x$-velocity $(\mathrm{m} / \mathrm{s})$

$v=y$-velocity $(\mathrm{m} / \mathrm{s})$

$V=$ volume $\left(\mathrm{m}^{3}\right)$

$\alpha=$ thermal diffusivity of fluid $\left(\mathrm{m}^{2} / \mathrm{s}\right)$

$\beta=$ pore to throat size ratio $\left(=H /\left(H-D_{y}\right)\right)$

$\varepsilon=$ porosity

$\nu=$ kinematic viscosity of fluid $\left(\mathrm{m}^{2} / \mathrm{s}\right)$

$\rho=$ density of fluid $\left(\mathrm{kg} / \mathrm{m}^{3}\right)$

\section{References}

[1] Nakayama, A., 1995, PC-Aided Numerical Heat Transfer and Convective Flow, CRC Press, Boca Raton, FL.

[2] Kaviany, M., 1995, Principles of Heat Transfer in Porous Media, 2nd ed., Springer-Verlag, New York.

[3] Kim, S. Y., Koo, J. M., and Kuznetsov, A. V., 2001, "Effect of Anisotropy in Permeability and Effective Thermal Conductivity on Thermal Performance of an Aluminium Foam Heat Sink," Numer. Heat Transfer, Part A, 40(1), pp. 21-36.

[4] Whitaker, S., 1999, The Method of Volume Averaging, Kluwer Academic, Dordrecht, The Netherlands.

[5] Quintard, M., and Whitaker, S., 1993, "One- and Two-Equation Models for Transient Diffusion Processes in Two-Phase Systems," Adv. Heat Transfer, 23, pp. 369-464.

[6] Quintard, M., and Whitaker, S., 1995, "Local Thermal Equilibrium for Transient Heat Conduction: Theory and Comparison With Numerical Experiments," Int. J. Heat Mass Transfer, 38(15), pp. 2751-2759.

[7] Kuwahara, F., Shirota, M., and Nakayama, A., 2001, "A Numerical Study of Interfacial Heat Transfer Coefficient in Two-Energy Equation Model for Convection in Porous Media," Int. J. Heat Mass Transfer, 44(6), pp. 1153-1159.

[8] Nakayama, A., Kuwahara, F., Umemoto, T., and Hayashi, T., 2002, "Heat and Fluid Flow Within an Anisotropic Porous Medium," ASME J. Heat Transfer, 124(4), pp. 746-753.

[9] Saito, M. B., and de Lemos, M. J. S., 2005, "Interfacial Heat Transfer Coefficient for Non-Equilibrium Convective Transport in Porous Media," Int. Commun. Heat Mass Transfer, 32(5), pp. 666-676.

[10] Saito, M. B., and de Lemos, M. J. S., 2006, "A Correlation for Interfacial Heat Transfer Coefficient for Turbulent Flow Over an Array of Square Rods," ASME J. Heat Transfer, 128(5), pp. 444-452.

[11] Lopez Penha, D. J., Stolz, S., Kuerten, J. G. M., Nordlund, M., Kuczaj, A. K., and Geurts, B. J., 2012, "Fully-Developed Conjugate Heat Transfer in Porous Media With Uniform Heating," Int. J. Heat Fluid Flow, 38, pp. 94-106.

[12] Gamrat, G., Favre-Marinet, M., and Le Person, S., 2008, "Numerical Study of Heat Transfer Over Banks of Rods in Small Reynolds Number Cross-Flow," Int. J. Heat Mass Transfer, 51(3-4), pp. 853-864.

[13] Alshare, A. A., Strykowski, P. J., and Simon, T. W., 2010, "Modeling of Unsteady and Steady Fluid Flow, Heat Transfer and Dispersion in Porous Media Using Unit Cell Scale," Int. J. Heat Mass Transfer, 53(9-10), pp. 2294-2310.

[14] Teruel, F. E., and Diaz, L., 2013, "Calculation of the Interfacial Heat Transfer Coefficient in Porous Media Employing Numerical Simulations," Int. J. Heat Mass Transfer, 60, pp. 406-412.

[15] Petrasch, J., Meier, F., Friess, H., and Steinfeld, A., 2007 "Tomography Based Determination of Permeability, Dupuit-Forchheimer Coefficient, and Interfacial Heat Transfer Coefficient in Reticulate Porous Ceramics," Int. J. Heat Fluid Flow, 29(1), pp. 315-326.

[16] Nakayama, A., Kuwahara, F., and Hayashi, T., 2004, "Numerical Modeling for Three-Dimensional Heat and Fluid Flow Through a Bank of Cylinders in Yaw,” J. Fluid Mech., 498, pp. 139-159.

[17] Kim, S. Y., and Kuznetsov, A. V., 2003, "Optimization of Pin-Fin Heat Sinks Using Anisotropic Local Thermal Nonequilibrium Porous Model in a Jet Impinging Channel," Numer. Heat Transfer, Part A, 44(8), pp. 771-787.

[18] Liu, D., and Garimella, S. V., 2005, "Analysis and Optimization of the Thermal Performance of Microchannel Heat Sinks," Int. J. Numer. Methods Heat Fluid Flow, 15(1), pp. 7-26.

[19] Ozgumus, T., Mobedi, M., and Ozkol, U., 2014, "Determination of Kozeny Constant Based on Porosity and Pore to Throat Size Ratio in Porous Medium With Rectangular Rods," Eng. Appl. Comput. Fluid Mech., 8(2), pp. 308-318.

[20] Özgümüs, T., Mobedi, M., Özkol, Ü., and Nakayama, A., 2013, "Thermal Dispersion in Porous Media-A Review on the Experimental Studies for Packed Beds," ASME Appl. Mech. Rev., 65(3), p. 031001.

[21] Hsu, C. T., 2000, "Conduction in Porous Media," Handbook of Porous Media, K. Vafai, ed., CRC Press, Boca Raton, FL.

[22] Patankar, S., 1980, Numerical Heat Transfer and Fluid Flow, Hemisphere Publishing Corp., New York. 